\title{
Experiments with Radiated Interference from In-Home Power Line Communication Networks
}

\author{
Brad Zarikoff* and David Malone ${ }^{\dagger}$ \\ Hamilton Institute, National University of Ireland Maynooth, Ireland \\ \{Brad.Zarikoff*, David.Malone $\left.{ }^{\dagger}\right\} @$ nuim.ie
}

\begin{abstract}
Power line communication (PLC) technology is at a tipping point and may become ubiquitous as a home networking technology. However, there are concerns regarding RF interference between PLC networks and other radio operators. It is imperative that the interference dynamics of PLC systems be fully understood in advance of any large-scale roll-out. In this work, we experiment with two interference scenarios: notch-infilling due to non-linear mixing and mitigation of radiated emissions using RF chokes. In our controlled test bed environment, we measure RF emissions from the power line devices in the $1 \mathrm{MHz}$ to $30 \mathrm{MHz}$ range. Through the results and simulation, we demonstrate that the effect of non-linear devices may be less of a concern than initially thought, and that simple methods can be employed to control local RF interference.
\end{abstract}

\section{INTRODUCTION}

With the recent acceptance of the IEEE P1901 Draft power line communications (PLC) standard [1], the networking community can expect to see an increase in the availability of commodity PLC hardware, and thus PLC-based home and small office networks. However, with an increase in active networks will come an associated increase in radiated emissions.

It is well-known that an unbalanced $50 / 60 \mathrm{~Hz}$ transmission line will become an effective transmission antenna for signals in the 1-30 MHz spectrum used in PLC networks [2], [3]. Although the effective radiated power from such signals will likely be low enough not to be of interest to systems outside of the local area [4], [5], local users may still have reason to complain. Not everyone shares this opinion however, and some concern has been raised over global spectrum pollution. The most vocal critics of this local interference are amateur radio enthusiasts, who are likely to live in urban and suburban areas and operate in close proximity to PLC devices (shortwave listeners may also be affected, but may not be aware as to the cause of poor reception). In response to their concerns, the PLC standards groups have implemented notches for the amateur radio bands. However, there is still the broad belief that such approaches fail due to lax certification procedures and/or additional network-based effects.

This work presents two experiments in the area of radiated PLC interference. The first experiment focuses on the networkbased effect of non-linear mixing. This phenomenon results from non-linear mixing between PLC signals and non-linear

The authors wish to acknowledge funding support from the Irish HEA PRTLI Cycle 4 FutureComm (http://futurecomm.tssg.org) programme and SFI grant $07 / \mathrm{SK} / \mathrm{I} 1216 \mathrm{a}$.

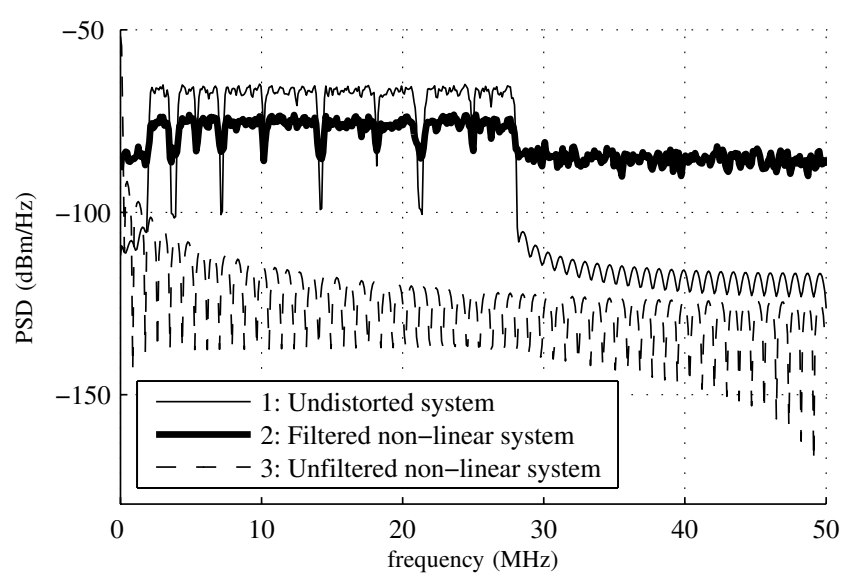

Fig. 1. Power spectral density (PSD) using Welch's method. Simulation comparison between 1) a linear network, 2) a $50 \mathrm{~Hz}$-filtered non-linear network, and 3) an unfiltered non-linear system. Model details are shown in Fig. 2. The non-linear model is given in (2), the PLC signal has peak power of $100 \mathrm{~mW}$ on a $50 \Omega$ line, and the $50 \mathrm{~Hz}$ signal is $240 \mathrm{~V}$.

devices (NLDs) sharing the same mains [6]. The result is thought to result in infilling of the PLC notches (e.g., compare trace 1 and 2 in Fig. 1). The second experiment focuses on passive ways of reducing PLC interference. The rationale is that unwanted RF interference (RFI) might be addressed by installing RF chokes on parts of the mains system most likely to radiate. Using RF chokes as a low-pass filter is a technique familiar to radio enthusiasts for RFI mitigation and may reduce RF emissions of PLC signals via the unbalanced in-ceiling loop circuit.

\section{BACKGROUND}

Our experiments in PLC do not contend to side with any party in this debate, but more to bring in a network-centric point of view. This specific work is a result of our discussions with amateur radio groups and national regulatory bodies.

The interference issues behind in-home PLC networks have been discussed for more than a decade [7]. Amateur radio groups are concerned that the transmission of communication signals via such a non-shielded system will interfere with their sensitive wireless systems. The unshielded power line network acts as an antenna (albeit an unintentional one) for the 1-30 $\mathrm{MHz}$ PLC signals. As a result, PLC networks may impact neighbouring radio amateurs. 


\begin{tabular}{|c|c|}
\hline Band & Channel $(\mathrm{kHz})$ \\
\hline \hline 160 metre amateur band & $1710-2000$ \\
\hline 80 metre amateur band & $3500-4000$ \\
\hline 60 metre amateur band & $5300-5450$ \\
\hline 40 metre amateur band & $7000-7300$ \\
\hline 30 metre amateur band & $10100-10150$ \\
\hline 20 metre amateur band & $14000-14350$ \\
\hline 17 metre amateur band & $18068-18168$ \\
\hline 15 metre amateur band & $21000-21450$ \\
\hline 12 metre amateur band & $24890-24990$ \\
\hline 10 metre amateur band & $28000-29700$ \\
\hline
\end{tabular}

TABLE I

Notches for HomePlug AV [10] AND IEEE 1901 [1] STANDARds.

Further, there was initial confusion as to what interference levels are applicable to PLC devices. CISPR is a part of the International Electrotechnical Commission (IEC) that sets standards for the control of electromagnetic interference. In the draft CISPR 22 document CISPR/I/89/CD, a two-tiered approach to PLC devices was proposed [8], where PLC devices were permitted a more lenient emissions mask. The justification for this was that PLC devices use the power line network as a communications medium, and are not emitting spurious emissions but controlled and conditioned signals. Thus a separate level should apply to communicating PLC devices. This approach does nothing to protect the sensitive long-range receivers operating within the same bands, and so this draft was not accepted; however, it does typify the uncertainty of certification groups on how to deal with PLC signals. New CISPR recommendations have been made since in the hopes that a consensus can be formed.

In the face of these issues, there are still numerous PLC devices in use today, which may or may not be causing issues for amateur radio users. It is our opinion that the interference issues are still not well understood. For the issue of non-linear mixing, there appears to be little academic investigative work. There have been experiments that attempt to illustrate the phenomenon [8], although as we point out later, we are cautious of the conclusions. For RF emissions via unbalanced loops, we have not found any published work on prior experimentation, although there is some experimental and simulation work for emissions of an entire house [9].

\section{POWER LINE RF INTERFERENCE}

\section{A. Notch Infilling}

The amateur radio community has expressed concern over the expansion of power line communication networks. At the onset, the main concern was direct interference between the PLC and the radio receivers, as they overlap in a number of bands (Table I). As a result, power line standards require notching at the above frequencies, to a level down to $80 \mathrm{dBm} / \mathrm{Hz}$ for the transmit spectrum. Note that even in the presence of notches, the radio receiver band selection filters play an important role in determining whether PLC transmissions will cause interference.
However, PLC signals may be subject to non-linear distortion when they are incident on other power line-centric devices. This may result in infilling, with the products falling into notch bands [6]. Some investigation into the effects of this distortion on PLC has been done [8], where a limiter is used as a non-linear device (NLD). The discussion in [8] suggests that while the presented results are an artefact of the measurement system, it is relevant to PLC since domestic power line networks are highly likely to include NLDs in the form of switched-mode power supplies or lighting dimmers.

To look deeper into the NLD notch-filling effect, we can compare the power line circuit with that of a standard RF mixer component. The mixer is a NLD used to modulate a message onto a carrier signal: the output of the mixer contains $n^{\text {th }}$ order products such that frequency components appear at multiples of the sum and difference of the original message and carrier frequencies. For example, take a single, phase modulated carrier (it is simple to generalize to the multi-carrier OFDM symbols used in PLC). At time $t$, with a phase modulated input message signal $f(t)$, and carrier $A_{c} \cos \left(2 \pi f_{c} t\right)$ at frequency $f_{c} \mathrm{~Hz}$, the output of the mixer is $s(t)$ and can be stated as

$$
s(t)=a_{0}+\sum_{i=1}^{N} a_{i}\left(f(t)+A_{c} \cos \left(2 \pi f_{c} t\right)\right)^{i},
$$

where $a_{i} \geq 0$ are parameters dependent on the specific NLD used for mixing. The conversion loss [11] is generally used to measure the amount of unwanted components of a RF mixer. Compared to the primary component, the products of the second and third order components can still be significant; the third order component ('third-order intermodulation products' in literature [12]) is a perennial problem in RF design. Such components are severe since they mix to the same frequency as the desired signal, and are particularly of concern in amplifiers, where the non-linearity comes from saturation. The experiments in Section V-A investigate the effect of such mixing in the lab environment.

As a means to estimate the impact of non-linear effects on the OFDM symbols used by PLC systems, we generated OFDM symbols in Matlab and looked at the power spectral density (PSD). Fig. 2 shows the three different models that correspond to the PSD traces of Fig. 1.

Our simple, filtered, non-linear model effectively captures the infilling effect observed in [8], which can be seen by comparing the notches in traces 1 and 2 . The results shown from [8] use a transient filter as a NLD, modeled here as

$$
s(t)=\max (\min (f(t),+1 V),-1 V,
$$

and placed between the mains-filtered LISN observation port and the spectrum analyser. The aim of the transient filter is to prevent power spikes at any frequency to arrive at the analyser input. However, the reliance on the LISN filter is unrealistic: in a domestic setting, such a filter would only exist within a device, and would likely be filtered by the power supply before any mixing occurred. Thus any signal remaining on the line 


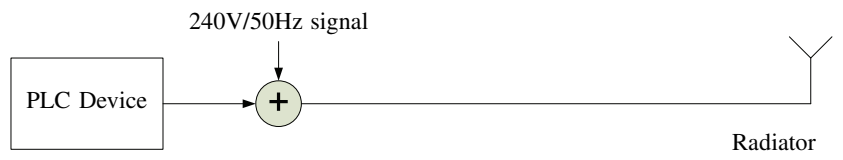

(a) Case 1: linear network

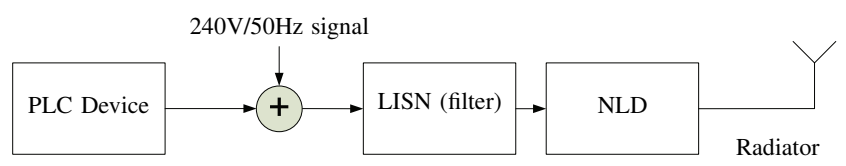

(b) Case 2: Non-linear network from [8]

$240 \mathrm{~V} / 50 \mathrm{~Hz}$ signal

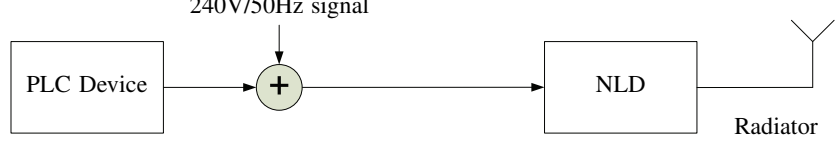

(c) Case 3: Our proposed non-linear network

Fig. 2. Three cases for RF emissions from a power line device. The non-linear device (NLD) serves as a mixer for the PLC signals, while the line impedance stabilization network (LISN) is a standard piece of equipment used in EMI testing. Case 1 is a simple linear model that serves as a baseline. Case 2 is a representation of the non-linear model used in experiments in [8] (we model it with (2)). Case 3 is what we propose as a more realistic non-linear model.

would include the $240 \mathrm{~V}$ mains signal. Trace 3 demonstrates the case where the non-linear operation of the transient limiter is used in the absence of the initial filter. The PLC signal is almost indistinguishable due to the saturation caused by the $240 \mathrm{~V} / 50 \mathrm{~Hz}$ signal.

\section{B. RF Emissions}

Additionally, there is also interference between PLC signals and signals that are not subject to notching (i.e. FM broadcast signals). Such problems have been noted in studies by the $\mathrm{BBC}$ [13]. As the propagation characteristics of PLC networks in the home are known [2], as are the mechanisms behind them (namely unbalanced, non-symmetric loops [14]), this does not come as a surprise. However, it is clearly a problem that cannot be addressed in the short term by the standards groups themselves. We suggest the simple solution of filtering of the mains cables via ferrite beads (chokes), and provide experimental verification that such a simple solution does in fact reduce the PLC signal transmission in unbalanced loops. These experiments are detailing is Section V-B.

\section{Test Bed Setup}

The power line test bed used for the following experiments contains two PLC devices. The PLC devices are made up of single-board Soekris computers with Devolo dLAN 200 AVminiPCI cards (a detailed description of the PLC devices is provided in [15]). Fig. 3 shows a logical schematic of our test bed. It shows the Soekris boxes with associated PLC cards, a connector block that serves as the communication medium, a spectrum analyser, a UPS used to isolate our test bed from the rest of the mains, a plugboard for our NLD experiments, and a $10 \mathrm{~m}$ ceiling loop for our emissions experiments. To couple the spectrum analyser to the medium we used an antenna; since our experiments focus on a relative difference in RF emissions of the PLC network, absolute measurements are not a concern.

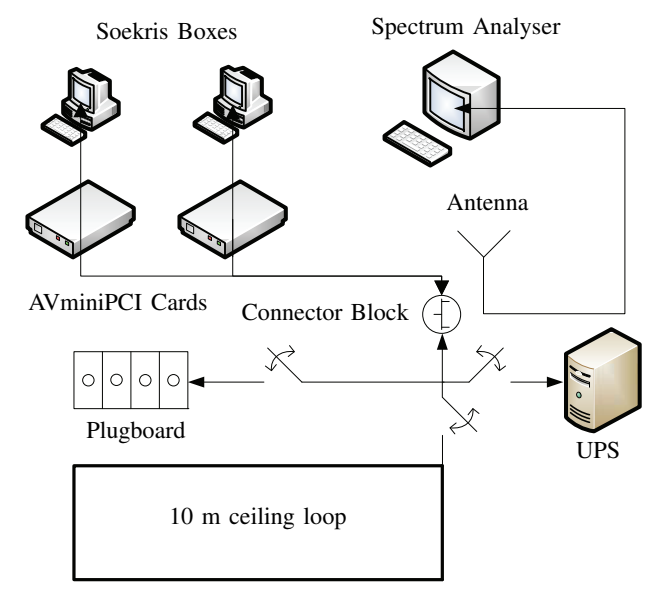

Fig. 3. Test bed logical schematic. The ceiling loop is connected in such a way as to unbalance the connector block network.

Further, since the PLC card is powered from the miniPCI bus, we can choose if we want to connect the PLC communication medium to the mains. This gives us control over the medium, and also allows us to monitor the physical channel directly. In this paper, we have used standard Devolo coupling devices to connect each of the miniPCI cards to the connector block.

For measuring the RF emissions of the test bed, a spectrum analyser was connected to our sensing antenna. Our measurement settings are provided in Table II. Note that the noise floor of a high-quality radio will be lower than in our tests. Our tests indicate that the spectral mask of the Devolo miniPCI cards was set to restrict sub-carriers to below $28 \mathrm{MHz}$, as per the HomePlug AV and IEEE 1901 standards. For the antenna, we used either a $4 \mathrm{~cm}$ diameter, two-turn unmatched loop antenna [16], or a broadband dipole antenna. Neither antenna was characterised or tuned to our band of interest. However, as we are only using it as a receive antenna for measuring changes in PSD, it is satisfactory.

We endeavored to create as clean and simple an environment as feasible in order to accurately measure the effect of each of the experiments. For the powered power line necessary for the infilling experiments, we used an APC Smart-UPS 750 power supply to clean up the mains signal and prevent additional distortion. This device makes use of a sinewave $50 \mathrm{~Hz} \mathrm{AC}$ signal, rather than a waveform step approximation common with other models. In this manner, we could operate either via a filtered mains signal or via the battery backup.

\section{EXPERIMENTAL OBSERVATIONS}

\section{A. Notch Infilling}

In Fig. 1, we saw that the details of the NLDs could have a significant impact on the PSD. In this section, we give results of experiments with common household devices: notebook computers (an Asus EeePc and Fujitsu Lifebook), a compact fluorescent (CFL) bulb, and $60 \mathrm{~W}$ incandescent bulb connected through an off-the-shelf dimmer switch (Crabtree CRO 1W400). All were connected via a plug-board which was directly wired to our switch box, as noted in Section IV. 


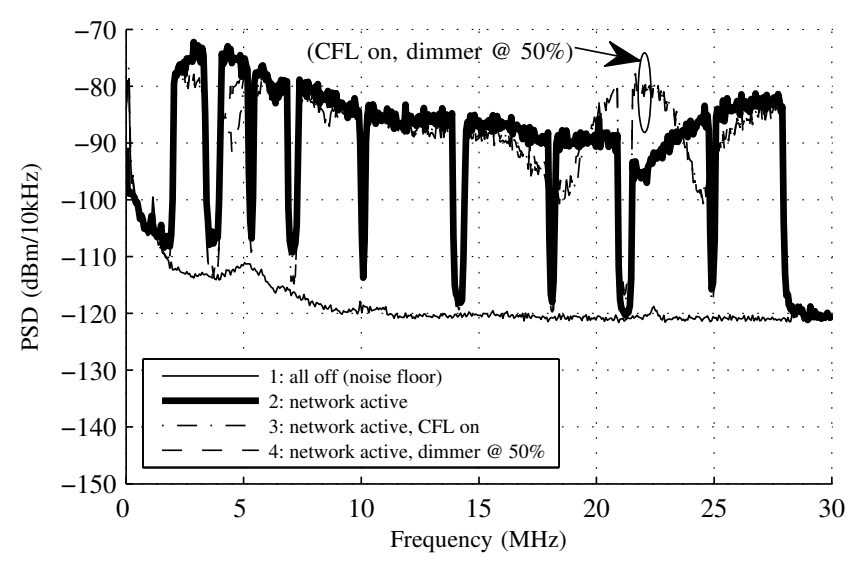

Fig. 4. Power spectral density (PSD) captured at connector block. 'Network active' refers to a running iperf test between PLC devices. The four traces correspond to the spectrum with 1) all devices powered down, 2) the network active, 3) the network active, compact fluorescent bulb on, and 4) the network active, a compact fluorescent bulb on, a dimmer operating an incandescent bulb at $50 \%$.

Our goal in this experiment was to verify if intermodulation distortion is measurable via the notch depth.

Our devices are based on the HomePlug AV standard [10], which use OFDM symbols in the frequency range from 2 $\mathrm{MHz}$ to $28 \mathrm{MHz}$ and an output PSD limit of $-50 \mathrm{dBm} / \mathrm{Hz}$. The transmit spectrum mask requires that the notched PSD is limited to $-80 \mathrm{dBm} / \mathrm{Hz}$, a total of $30 \mathrm{~dB}$ less than the spectrum in the passband. Therefore, we expect to see spectrum notches of at least $30 \mathrm{~dB}$.

Fig. 4 shows a full span spectrum capture of the test bed with the in-ceiling loop disconnected. There are four traces shown: 1) with the devices powered off ('all off'), 2) with the devices powered and transmitting data ('network active'), 3) with the devices powered, transmitting data and the CFL bulb powered ('network active, CFL-on'), and 4) with the devices powered, transmitting data and the dimmer switch at 50\% ('network active, dimmer at 50\%'). Additional tests using notebook PC power supplies were also done with similar results. The spectrum notches of $>30 \mathrm{~dB}$ are clearly visible. A change in the whole spectrum when the NLDs are powered is evident, particularly around $4 \mathrm{MHz}$ and $20 \mathrm{MHz}$. We attribute this to a change in the network impedance, and thus the radiation pattern of the power line network. In the 15 metre amateur band in fact, it appears that the notch is filled with intermodulation components. However, on a closer inspection, it is clear that the notch itself is still at the same level compared to the un-notched signal to either side. Fig. 5 provides a closeup view, and illustrates that this apparent notch-filling is really an artefact of the impedance change.

In order to gather concrete evidence of NLDs filling in PLC spectral notches, extended tests are required. However, our experiments point to the possibility that mixing may be benign in a domestic setting (with CFL, dimmers and PC power supplies). The observation of such mixing may in fact be a

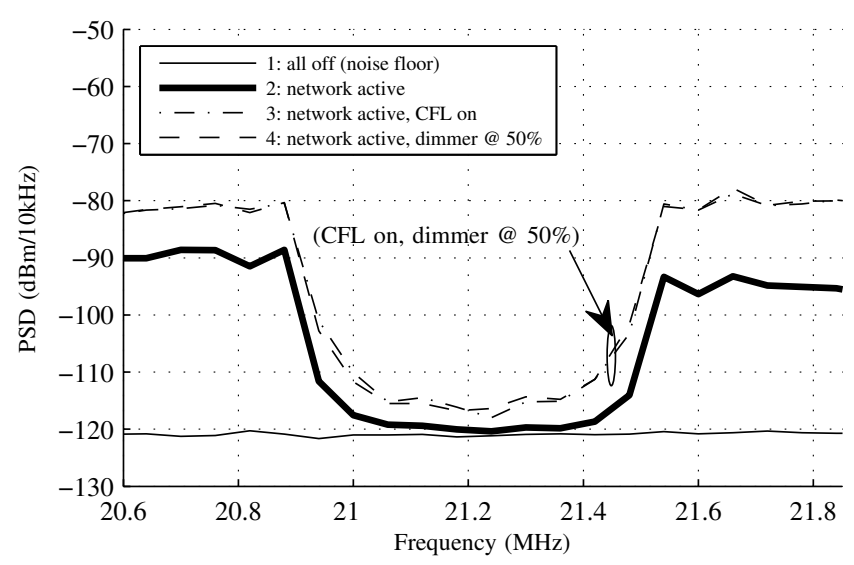

Fig. 5. Power spectral density (PSD) captured at connector block. Magnified version of Fig. 4 in the range [20.6,21.85] MHz, showing the effect of the impedance change on the notch.

product of impedance changes on the power line network. ${ }^{1}$

\section{B. External Interference}

In order to deal with in-home RF interference caused by power line devices, we explore the idea of RF chokes to act as a PLC signal filter. A significant factor in power line RF emissions is unbalanced or non-symmetric cable loops within the home [9]. In Europe and North America, such loops are commonly used to power overhead lights. When a light switch is engaged, and a power line network is present, then these loops turn into effective radiators. Our approach is simple: attach RF chokes to the base of such a loop and measure the effect on the radiated PLC signal.

To focus on the power radiated from our test bed, we isolated our experiment from the primary mains in the lab. This limited the possibility that mains loops elsewhere were acting as radiators. The results are shown in Fig. 6. There are three traces shown: 1) with the devices powered off and the loop disconnected, 2) with the devices powered on and transmitting data, ('network active') and the loop connected, and 3) with the devices powered on and transmitting data, and the loop connected with three ferrite RF chokes at each connection point to the connector block. The chokes were off-the-shelf components: Maplin HEM3012 chokes, with impedances of $164 \Omega$ at $25 \mathrm{MHz}$ and $255 \Omega$ at $100 \mathrm{MHz}$. In order to limit near-field effects, we situated our antenna in the next room, 6 $\mathrm{m}$ from the loop. The variation in the baseline measurements are much more varied than in the notching experiments, since the dipole antenna is more effective at capturing transmissions in the HF 3-30 MHz band.

Although the spectrum is more distorted than the near-field measurements in the previous section, the presence of a PLC signal is clear from the difference between the 'all off' and the 'no ferrites' traces: in particular, the 30 metre amateur band

\footnotetext{
${ }^{1}$ Subsequent tests done with the Fujitsu notebook have produced evidence of notch infilling. The observations include infilling of upwards of $5 \mathrm{~dB}$ on select notches, while other notches are untouched.
} 


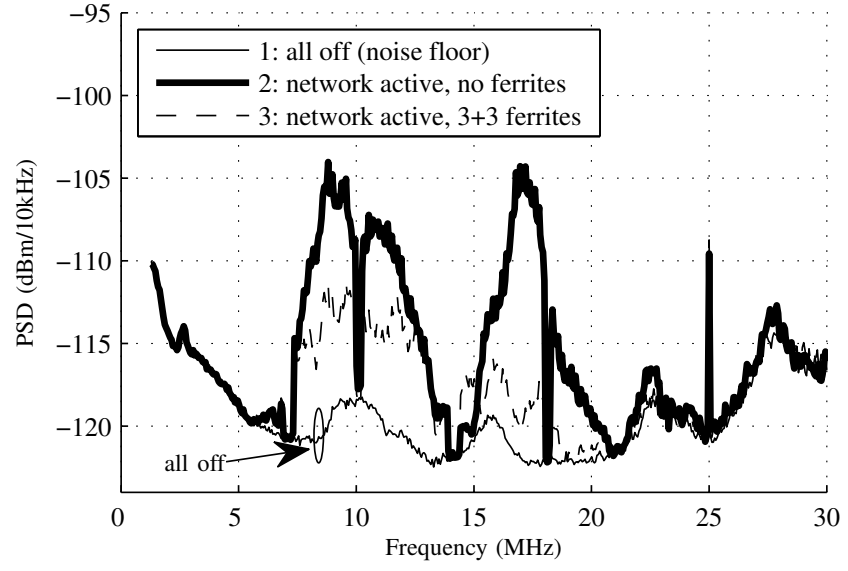

Fig. 6. Power spectral density (PSD) captured in adjacent room $(>6 \mathrm{~m}$ away). 'Network active' refers to a running iperf test between PLC devices. The three traces correspond to the spectrum with 1) all devices powered down, ceiling loop disconnected, 2) the network active, ceiling loop connected, no ferrites used, and 3) the network active, ceiling loop connected, and 3 ferrites attached to either end of the ceiling loop. For all traces, the switch block was un-powered.

\begin{tabular}{|c||l|l|l|l|l|}
\hline Setting & RBW & VBW & $f_{c}$ & Trace & Detector \\
\hline Value & $10 \mathrm{kHz}$ & $30 \mathrm{kHz}$ & $15 \mathrm{MHz}$ & Max-hold & $\begin{array}{l}\text { Positive } \\
\text { Peak }\end{array}$ \\
\hline
\end{tabular}

TABLE II

MEASUREMENT SETTINGS FOR THE ROHDE \& SCHWARZ FSL6 SPECTRUM ANALYSER USED FOR TESTS.

notch is clearly visible. After adding the ferrite chokes, a clear drop in the transmitted spectrum is visible, particularly from $7.5 \mathrm{MHz}$ to $12.5 \mathrm{MHz}$ and $15 \mathrm{MHz}$ to $18 \mathrm{MHz}$. In the notches, the signal level maintains the level or drops (note the 30 metre band notch). Of note, there are some parts of the trace where the RF chokes appear to accentuate the PLC RF emissions. We believe this is a result of impedance changes as commented on in the last section. In general however, with simple, untuned, off-the-shelf chokes, it is clear that emissions can be reduced. While this approach is far from ideal for the general consumer, it may be useful in certain circumstances. Further work is warranted on determining whether the elimination of all overhead loops within a home would substantially reduce the amount of PLC generated RF emissions, and on the use of tuned chokes.

\section{Unbalanced Feed to Non-linear Devices}

To combine the effects of an unbalanced loop and NLDs, we did tests with the in-ceiling loop using the non-linear dimmer switch. This is done particularly because NLDs themselves can act to unbalance the power line network. The results are not shown here. The notches did not appear to suffer from infilling, although because of the ambient noise and our outof-band dipole antenna, we could not get a satisfactory far-field picture of the notches (the strongest PLC signal captured was only $20 \mathrm{~dB}$ above the noise floor). While we cannot conclude that infilling did not occur, it was not discernable in our tests.

\section{CONCLUSION}

In-home PLC systems have not gained support from all spectrum users. In particular, amateur radio operators are concerned about in-band interference, even with PLC devices that use dedicated notching in amateur bands. The reasons for such concerns include non-linear mixing with other appliances attached to the power line network and general radiation of PLC signals. The experiments presented here have been done using a PLC test bed with commodity devices. First, we have shown that the non-linear mixing effect may not be as serious as first considered. Experimental evidence does not show infilling in the presence of a non-linear compact fluorescent bulb and dimmer switch, although subsequent testing has shown selective infilling using a notebook power supply unit. Second, we have demonstrated that simple methods may be sufficient for reducing PLC RF emissions. Using RF chokes, we have demonstrated a reduction of emissions on a scale of $8-15 \mathrm{~dB}$.

\section{ACKNOWLEDGEMENTS}

We acknowledge Brendan Minish for his insights into the contentious issue of PLC in the amateur radio community.

\section{REFERENCES}

[1] Broadband Over Power Lines PHY/MAC Working Group, "IEEE P1901/D4.01 Draft Standard for Broadband over Power Line Networks," IEEE, Tech. Rep., 2010.

[2] M. Ishihara, D. Umehara, and Y. Morihiro, "The Correlation between Radiated Emissions and Power Line Network Components on Indoor Power Line Communications," in IEEE Int. Symp. on Power Line Commun. and Its Applications (ISPLC), Apr. 2006, pp. 314 -318.

[3] R. Marshall, "Environmental Effects of the widespread deployment of high speed Power Line Communication," EMC Journal, no. 87, 2010.

[4] PA Consulting Group, "The Likelihood and Extent of Radio Frequency Interference from In-Home PLT Devices," Ofcom, Tech. Rep., Jun. 2010, http://bit.ly/o1II2Y.

[5] M. Zhang and W. Lauber, "Evaluation of the Interference Potential of PLC Systems," in IEEE Int. Symp. on Power Line Commun. and Its Applications (ISPLC), Apr. 2006, pp. 296 -301.

[6] "What is Power Line Technology," Ban Power Line Technology, Tech. Rep., May 2011, http://bit.ly/qXR6O3.

[7] B. Denton, D. Fifield, S. Gardner, E. Hare, and B. Markwalter, "Joint Test Report," HomePlug Powerline Alliance/American Radio Relay League, Tech. Rep., Jan. 2001.

[8] T. Williams, "RF Emissions of Powerline Ethernet Adaptors," EMC Journal, no. 82, 2009.

[9] R. Vick, "Radiated emission caused by in-house PLC systems," in IEEE Int. Symp. on Power Line Commun. and Its Applications (ISPLC), Apr. 2001.

[10] HomePlug Powerline Alliance, "HomePlug AV White Paper," 2005, http://bit.ly/ptpeN1.

[11] D. M. Pozar, Microwave and RF Design of Wireless Systems. New York: John Wiley \& Sons, Inc., 2001.

[12] W. C. Babcock, "Intermodulation interference in radio systems," Bell Systems Tech. Jour., pp. 63-73, 1953.

[13] M. Waddell and J. Stott, "VHF emissions from PLT devices," British Broadcasting Corporation, Tech. Rep., Mar. 2011, http://bbc.in/eSPWew.

[14] V. Arnautovski-Toseva, K. El Khamlichi Drissi, and K. Kerroum, "Antenna mode currents and radiated emissions of in-door PLC line within wall structure," in IEEE Int. Symp. on Power Line Commun. and Its Applications (ISPLC), Apr. 2011, pp. 231 -236.

[15] B. Zarikoff and D. Malone, "Construction of a PLC test bed for network and transport layer experiments," in IEEE Int. Symp. on Power Line Commun. and Its Applications (ISPLC), Apr. 2011, pp. 135-140.

[16] N. Nikolova, "Lecutre Notes: Loop Antennas," McMaster University, Canada, Tech. Rep., 2010, http://bit.ly/oImt8E. 